\title{
Long Branching in Poly(vinyl acetate) and Poly(vinyl alcohol) V. Determination of the Long-Chain Branching in Poly(vinyl alcohol).
}

\author{
Yotaro Morishima, Woo-sik Kim, ${ }^{*}$ and Shun-ichi Nozakura \\ Department of Polymer Science, Faculty of Science, \\ Osaka University, Toyonaka 560, Japan. \\ (Received October 16, 1975)
}

\begin{abstract}
Long branching in poly(vinyl alcohol) (PVA) was quantitatively determined from measurements of the intrinsic viscosity and gel-permeation chromatography of derived poly(vinyl acetate) (PVAc) according to Kurata's method. The average number of branch points per unit molecular weight, $\lambda_{3}$, was observed to lie in the range of $10^{-6}-10^{-5}$ for PVA obtained from PVAc polymerized at $60^{\circ} \mathrm{C}$ in bulk. The tendency for the values of $\lambda_{3}$ to increase with the increase in the conversion of polymerization of the parent polymer was found to be in close agreement with that expected from the kinetic equation proposed in our previous paper.

KEY WORDS Long Branching / Poly(vinyl alcohol) / Poly(vinyl acetate)/ Gel-Permeation Chromatography / Intrinsic Viscosity / Branching Parameter /
\end{abstract}

In previous papers ${ }^{1,2}$ the formation of longchain branches in poly(vinyl alcohol) (PVA) was kinetically analyzed. It was clarified that the long branches in PVA are exclusively formed by the chain transfer to polymer and that the amount of the branch increases as the conversion increases and as the polymerization temperature rises. As a result, the amount of long branches in PVA derived from bulk polymerization of vinly acetate at $60^{\circ} \mathrm{C}$ is predictable from kinetic equations. However, the quantitative determination of long-chain branches for a given PVA specimen has not been reported so far. A method has been proposed for estimating the degree of branching from the elution curve of GPC and the intrinsic viscosity of unfractionated samples. Drott, et al., ${ }^{3,4}$ determined long branches in polyethylene using this method. Kurata, et al., ${ }^{5-7}$ also reported the same method independently, and discussed the limit of reliability by showing an example of the application to branched polystyrene. In the present paper, the amount of long-chain branches in PVA was determined by means of Kurata's method, ${ }^{5-7}$ and the results were compared with those expected

* Leave of absence from Department of Polymer Science, Kyungpook National University, Korea. from the kinetic scheme proposed in our previous work. ${ }^{2}$

\section{EXPERIMENTAL}

\section{Materials}

Vinyl acetate was purified according to the previous paper. ${ }^{2}$ Tri- $n$-butylborane was distilled under reduced pressure (bp, $90-91^{\circ} \mathrm{C} / 9 \mathrm{mmHg}$ ), and 2,2'-azobisisobutyronitrile (AIBN) was recrystallized from methanol. Benzene was dried over $\mathrm{CaCl}_{2}$ and distilled through a Widmer column twice. Methanol and tetrahydrofuran were distilled through a Widmer column.

Polymerization of Vinyl Acetate

Polymerization of vinyl acetate at $0^{\circ} \mathrm{C}$ was carried out in the following way. To $21.9 \mathrm{~g}$ of vinyl acetate and $8.1 \mathrm{~g}$ of methanol contained in a $50-\mathrm{m} l$ Shlenk tube kept at $0^{\circ} \mathrm{C}$ were added $0.23 \mathrm{ml}$ of tri- $n$-butylborane $(0.5 \mathrm{~mol} \%$ to monomer) and $1 \mathrm{~m} l$ of oxygen as initiators under an atmosphere of nitrogen. The polymerization was quenched at conversion lower than $10 \%$ by the addition of $1 \mathrm{ml}$ of $5-\%$ methanolic solution of hydroquinone. The contents were concentrated and poured into $n$-hexane to precipitate poly(vinyl acetate) (PVAc). The precipitated polymer was 
Long Branching in PVAc and PVA. V.

purified by reprecipitation into water from an acetone solution. The purified polymer was dried at room temperature in vacuo.

Bulk polymerizations at 60 and $90^{\circ} \mathrm{C}$ were carried out using AIBN as the initiator according to the previous paper. ${ }^{2}$

\section{Saponification and Reacetylation}

Saponification and reacetylation were carried out in accordance with a previous paper. ${ }^{2}$ The PVAc was fractionated by the partial precipitation method using the acetone-water system, and the fractionated PVAc was dissolved in benzene and recovered by a freeze-drying technique.

\section{Polystyrene}

Nine kinds of commercial polystyrene were used as standard polymers for GPC. They are anionically-polymerized samples with narrow distributions of molecular weight. Three samples with a molecular weight range of $2.0 \times 10^{6}-$ $5.5 \times 10^{6}$ were obtained from Toyo Soda Co., Ltd., and six samples with the range of $1.0 \times$ $10^{4}-2.0 \times 10^{6}$ were from Pressure Chemical Co., Ltd.

\section{Viscosity Measurement}

PVAc and standard polystyrene were dissolved in benzene at $45^{\circ} \mathrm{C}$. The viscosity of the benzene solution was measured with an Ubbelohde viscometer which has an upper bulb of $2 \mathrm{~m} l$ and the flow time of $140.8 \mathrm{sec}$ at $35^{\circ} \mathrm{C}$ for benzene. The intrinsic vicosity was determined from the combination of Huggins' plot and Fuoss-Mead's plot. Two independent measurements were made on each sample and the obtained values were averaged, the values of each run were practically equal.

\section{GPC Measurement}

PVAc and polystyrene were dissolved in tetrahydrofuran at $45^{\circ} \mathrm{C}$ and then the solutions were cooled to room temperature. All traces of insoluble particles in the solutions were removed by a Millipore filter with a pore size of $0.25 \mathrm{~m} \mu$ diameter. GPC measurements were carried out with a Toyo Soda High Speed Liquid Chromatograph HLC-807 under the following conditions: polymer concentration, $0.25 \mathrm{wt} \%$; flow rate, $1.8 \mathrm{ml} / \mathrm{min}$; temperature, $40^{\circ} \mathrm{C}$.

\section{RESULTS AND DISCUSSION}

Equations $1-6$ of Kurata's method ${ }^{5,6}$ were used to estimate the branching parameter $\lambda$ (the number of branch points per unit molecular weight of the polymer) from the intrinsic viscosities and GPC elution curves.

$$
\begin{gathered}
\log Q_{i}=C-D V_{i} \\
Q_{i}=[\eta]_{i} M_{i}=K M_{i}{ }^{1+A} G\left(\lambda_{4}, M_{i}\right) \\
{[\eta]=\Sigma_{i} f_{i}[\eta]_{i}=K \Sigma_{i} f_{i} M_{i}{ }^{A} G\left(\lambda_{4}, M_{i}\right)} \\
G\left(\lambda_{4}, M_{i}\right)=\left[\left(1+\lambda_{4} M_{i} / 6\right)^{1 / 2}+(4 / 3 \pi) \lambda_{4} M_{i}\right]^{-0.3}
\end{gathered}
$$

Here, $Q_{i}$ is the product of the intrinstic viscosity $[\eta]_{i}$ and the molecular weight $M_{i} . \quad C$ and $D$ are constants in a universal calibration curve of GPC. $V_{i}$ is the elution count, $K$ and $A$ are the Mark-Houwink constants for linear polymers, $\lambda_{4}$ is the branching parameter for a randomly branched polymer with tetrafunctional branch points, and $f_{i}$ is the weight fraction of molecules at the elution count $V_{i}$. In this study, $f_{i}$ was read on the elution curve at 0.5 intervals in count number in accordance with the literature. ${ }^{5,7} G\left(\lambda_{4}, M\right)$ is defined as the ratio of the intrinsic viscosity of branched polymer $[\eta]_{b}$ to that of the linear polymer $[\eta]_{l}$ of the same molecular weight and is theoretically correlated with $\lambda_{4}$ and $M$ by eq 4 .

$$
[\eta]_{b} /[\eta]_{l}=G\left(\lambda_{4}, M_{i}\right)
$$

In order to calculate the branching parameter from these equations, the computer program for the iterative method proposed by Kurata ${ }^{5,7}$ was used: that is, $\lambda_{4}$ is determined by the gradual increases of $\lambda_{4}$ starting from $\lambda_{4}=0$ until the calculated viscosity agrees with the experimental viscosity. Since the long-chain branch in PVA is formed by the mechanism of polymer transfer, ${ }^{1,2}$ PVA should have random branching with trifunctional branch points. The branching parameter for a randomly branched polymer with trifunctional branch points, $\lambda_{3}$, can be approximately calculated from $\lambda_{4}$ according to eq $6 .^{6}$

$$
\lambda_{3}=2.67 \lambda_{4}
$$

The first step in this analysis is the determination of the constants $C, D, K$, and $A$. The universal calibration curve measured for standard 
Y. Morishima, W. KIM, and S. Nozakura

Table I. Analysis of GPC elution curve for fractionated linear PVAc ${ }^{\text {a }}$

\begin{tabular}{ccccccc}
\hline $\begin{array}{c}\text { Sample } \\
\text { code }\end{array}$ & $\begin{array}{c}M_{n \lambda=0,} \\
\times 10^{-5}\end{array}$ & $\begin{array}{c}M_{w \lambda=0}, \\
\times 10^{-5}\end{array}$ & $\frac{M_{w \lambda=0}}{M_{n \lambda=0}}$ & $\begin{array}{c}{[\eta]_{\mathrm{obs}},} \\
\mathrm{d} l / \mathrm{g}\end{array}$ & $\begin{array}{c}{[\eta] \lambda=0,} \\
\mathrm{~d} l / \mathrm{g}\end{array}$ & $\frac{[\eta]_{\mathrm{obs}}}{[\eta]_{\lambda=0}}$ \\
\hline A-1 & 2.54 & 5.32 & 2.09 & 1.48 & 1.46 & 1.02 \\
A-2 & 2.26 & 4.60 & 2.04 & 1.36 & 1.33 & 1.01 \\
A-3 & 1.93 & 3.22 & 1.67 & 1.06 & 1.07 & 0.99 \\
A-4 & 1.66 & 2.48 & 1.49 & 0.90 & 0.90 & 1.00 \\
A-5 & 1.17 & 1.66 & 1.42 & 0.71 & 0.69 & 1.03 \\
A-6 & 0.63 & 0.98 & 1.56 & 0.49 & 0.47 & 1.03 \\
\hline
\end{tabular}

a Polymerized at $0^{\circ} \mathrm{C}$ in methanol, saponified, and reacetylated.

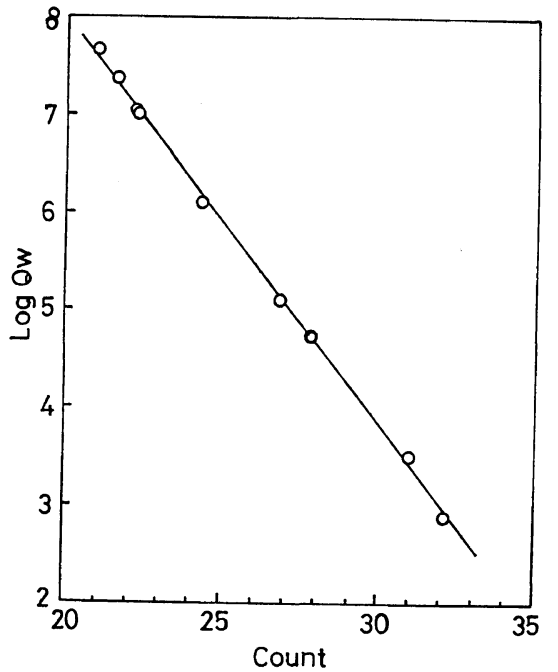

Figure 1. GPC universal calibration curve.

polystyrene is shown in Figure 1 and the constants $C$ and $D$ in eq 1 were determined by the leastsquares method.

$$
\log Q_{w}=16.22-0.413 V
$$

Here, $Q_{w}$ is the product of [ $\left.\eta\right]$ and $M_{w}$. Data for the Mark-Houwink constants, $K$ and $A$, have been reported for PVAc polymerized under various conditions. Since we need $K$ and $A$ for linear PVAc, i.e., PVAc prepared at a low conversion and a low temperature, ${ }^{2}$ the values reported by Hobbs, et $a .^{8}{ }^{8}$ seem appropriate to our purpose. In their report, vinyl acetate was polymerized at $-19^{\circ} \mathrm{C}$ with $\mathrm{AIBN}$ as a photosensitizer. PVAc was fractionated by the partial precipitaion method and $M_{w}$ was determined by the light scattering method. The present authors recalculated the constants $K$ and $A$ from

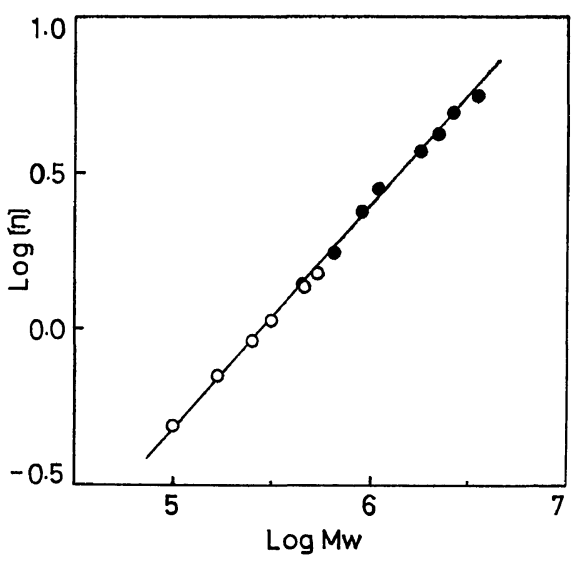

Figure 2. Relation between $M_{w}$ and intrinsic viscosity for PVAc polymerized at low temperature: $\bigcirc$, our data from GPC; 0 , Hobbs, et al.'s data.

Hobbs, et al.'s data ${ }^{8}$ by the least-squares method, yielding eq 8 .

$$
[\eta]=1.877 \times 10^{-4} M_{w}{ }^{0.686}
$$

In order to examine the reliability of this estimation, GPC and intrinsic viscosity measurements for PVAc, which is expected to be a linear polymer, were carried out. This PVAc was prepared under the conditions of $0^{\circ} \mathrm{C}$ and low conversion, saponified, reacetylated to eliminate hydrolyzable branches, and fractionated. The intrinsic viscosity as $\lambda=0$ and the molecular weight were computed, based on the GPC data, by the use of above values of $C, D, K$, and $A$. As seen in Table $I$, the ratios of the observed intrinsic viscosities ( $\left.[\eta]_{\mathrm{obs}}\right)$ to those calculated at $\lambda=0\left([\eta]_{\lambda=0}\right)$ lie in the range of $0.99-1.03$ (the error is about $\pm 3 \%$ ), Kurata, et al., ${ }^{5}$ suggested 
Long Branching in PVAc and PVA. V.

Table II. Branching parameters from the analysis of the GPC elution curve for reacetylated PVA derived from PVAc polymerized at 60 and $90^{\circ} \mathrm{C}$

\begin{tabular}{|c|c|c|c|c|c|c|c|c|c|c|}
\hline $\begin{array}{l}\text { Sample } \\
\text { code }\end{array}$ & $\begin{array}{l}\text { Fractional } \\
\text { conversion }\end{array}$ & $\begin{array}{c}\text { Run } \\
\text { no. }\end{array}$ & $\begin{array}{c}M_{n \lambda=0}=0 \\
\times 10^{-5}\end{array}$ & $\begin{array}{r}M_{w \lambda=0} \\
\times 10^{-5}\end{array}$ & $\begin{array}{r}{[\eta]_{\mathrm{obs}},} \\
\mathrm{d} l / \mathrm{g}\end{array}$ & $\begin{array}{c}{[\eta] \lambda=0,} \\
\mathrm{~d} l / \mathrm{g}\end{array}$ & $\frac{[\eta]_{\text {obs }}}{[\eta]_{\lambda=0}}$ & $\begin{array}{c}M_{n}, \\
\times 10^{-5}\end{array}$ & $\begin{array}{l}M_{w} \\
\times 10^{-5}\end{array}$ & $\begin{array}{l}\lambda_{3}, \\
\times 10^{6}\end{array}$ \\
\hline \multirow[t]{4}{*}{ B-1 } & 0.185 & 1 & 2.84 & 7.76 & 1.80 & 1.87 & 0.96 & 2.86 & 8.14 & 0.91 \\
\hline & & 2 & 2.85 & 7.32 & 1.80 & 1.81 & 0.99 & 2.86 & 7.37 & 0.16 \\
\hline & & 3 & 2.74 & 7.67 & 1.80 & 1.86 & 0.97 & 2.76 & 8.00 & 0.85 \\
\hline & & & & & & & & & av & $\overline{0.64}$ \\
\hline \multirow[t]{6}{*}{$\mathrm{B}-2^{\mathrm{a}}$} & 0.498 & 1 & 1.27 & 5.59 & 1.39 & 1.47 & 0.94 & 1.28 & 6.00 & 2.14 \\
\hline & & 2 & 1.58 & 5.59 & 1.39 & 1.50 & 0.93 & 1.60 & 6.10 & 2.96 \\
\hline & & 3 & 1.16 & 5.32 & 1.39 & 1.43 & 0.97 & 1.17 & 5.52 & 1.07 \\
\hline & & 4 & 1.23 & 5.41 & 1.39 & 1.45 & 0.96 & 1.23 & 5.68 & 1.42 \\
\hline & & 5 & 1.31 & 5.65 & 1.39 & 1.50 & 0.93 & 1.33 & 6.18 & 2.96 \\
\hline & & & & & & & & & av & 2.11 \\
\hline \multirow[t]{7}{*}{ B $-3^{a}$} & 0.677 & 1 & 1.40 & 5.46 & 1.36 & 1.45 & 0.94 & 1.42 & 5.93 & 2.56 \\
\hline & & 2 & 1.49 & 5.81 & 1.36 & 1.51 & 0.90 & 1.52 & 6.62 & 4.43 \\
\hline & & 3 & 1.57 & 5.65 & 1.36 & 1.50 & 0.91 & 1.60 & 6.36 & 4.30 \\
\hline & & 4 & 1.34 & 5.70 & 1.36 & 1.50 & 0.91 & 1.37 & 6.40 & 4.06 \\
\hline & & 5 & 1.26 & 5.52 & 1.36 & 1.46 & 0.93 & 1.27 & 6.03 & 2.80 \\
\hline & & 6 & 1.21 & 5.81 & 1.36 & 1.51 & 0.90 & 1.23 & 6.60 & 4.27 \\
\hline & & & & & & & & & av & 3.74 \\
\hline \multirow[t]{6}{*}{ B $-4^{a}$} & 0.791 & 1 & 1.38 & 5.41 & 1.32 & 1.44 & 0.91 & 1.41 & 6.04 & 3.74 \\
\hline & & 2 & 1.75 & 5.54 & 1.32 & 1.49 & 0.89 & 1.81 & 6.40 & 5.98 \\
\hline & & 3 & 1.32 & 5.19 & 1.32 & 1.41 & 0.94 & 1.33 & 5.64 & 2.83 \\
\hline & & 4 & 1.34 & 5.31 & 1.32 & 1.43 & 0.92 & 1.36 & 5.85 & 3.34 \\
\hline & & 5 & 1.67 & 5.44 & 1.32 & 1.47 & 0.92 & 1.71 & 6.18 & 5.07 \\
\hline & & & & & & & & & av & $\overline{4.19}$ \\
\hline $\mathrm{H}-1^{\mathrm{b}}$ & 0.792 & 1 & 0.91 & 2.83 & 0.79 & 0.92 & 0.86 & 0.95 & 3.44 & 16.02 \\
\hline $\mathrm{H}-\mathrm{1}^{\mathrm{b}}$ & 0.888 & 1 & 0.70 & 2.49 & 0.74 & 0.84 & 0.89 & 0.71 & 2.91 & 11.75 \\
\hline
\end{tabular}

a B series, samples polymerized at $60^{\circ} \mathrm{C}$.

b $\mathrm{H}$ series, samples polymerized at $90^{\circ} \mathrm{C}$.

that the accuracy for the estimation of intrinsic viscosity is within $5 \%$, which covers the error in the present study. Since the distribution function of the molecular weight is calculated in the course of this iterative method, various kinds of average-molecular weights $\left(M_{w}, M_{n}\right)$ can also be evaluated. The relation between $[\eta]_{\mathrm{obs}}$ and the computed $M_{w}$ was plotted in Figure 2, together with those from Hobbs, et al.'s data. ${ }^{8}$ Both plots lie on the straight line represented by eq 8 . These facts indicate that the constants $C, D, K$, and $A$ have been correctly determined.

Samples with long-chain branches which were polymerized at 60 and $90^{\circ} \mathrm{C}$ were analyzed; the results are shown in Table II. The molecular weights calculated at $\lambda=0$ are shown for reference. It is reasonable that these molecular weights are less than those calculated at $\lambda \neq 0$. Several runs of measurements were made on each sample and $\lambda_{3}$ was computed for cach individual run. The average values are shown in Table II. All the ratios, $[\eta]_{\mathrm{obs}} /[\eta]_{\lambda=0}$, are less than unity, while those of the samples polymerized at $0^{\circ} \mathrm{C}$ are substantially unity, as shown in Table I. Therefore, the samples polymerized at $60^{\circ} \mathrm{C}$ obviously have long-chain branches. The calculated values of $\lambda_{3}$ for sample B-1 in Table II may not be reliable, because the ratios are between 0.96 and 0.99 within the expected error of $\pm 3 \%$. The ratio, $[\eta]_{\text {obs }} /[\eta]_{\lambda=0}$, was observed to decrease with the increasing conversion of polymerization and the observed $\lambda_{3}$ for the samples other than B-1 should be reliable. 


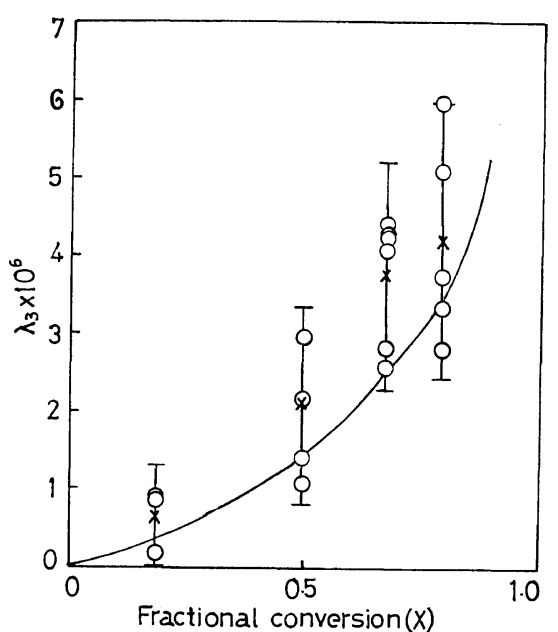

Figure 3. Calculated and observed branching parameter $\lambda_{3}$ as a function of fractional conversion $x$ : $\mathrm{x}$, averaged value; $\bigcirc$, individual value for each run; vertical bars represent the error range of $\lambda_{3}$.

Table II shows that the amount of long-chain branches of the samples polymerized at $90^{\circ} \mathrm{C}$ and to high conversions is very high in comparison with that of the samples polymerized at $60^{\circ} \mathrm{C}$ and to low conversions. This is just what is expected from the kinetic study. ${ }^{2}$

Let us compare the measured $\lambda_{3}$ with that expected from kinetics. The kinetic equation in a previous paper $^{2}$ can be related to $\lambda_{3}$ by eq 9.

$$
\lambda_{3}=\frac{[\mathrm{NBg}]}{x[\mathrm{M}]_{0}} \frac{1}{M}=\frac{C_{p, 2}}{M}\left(\frac{1}{x} \ln \frac{1}{1-x}-1\right)
$$

Here, $[\mathrm{NBg}]$ is the concentration of nonhydrolyzable branching points in molar, $x$ is the fractional conversion, $[\mathrm{M}]_{0}$ is the initial molar concentration of monomer, and $M$ is the molecular weight of monomer. $C_{p, 2}$ is the chain-transfer constant for the main chain of polymer, $3.03 \times 10^{-4}$ at $60^{\circ} \mathrm{C}$. Equation 9 gives the curve in Figure 3. The observed values of $\lambda_{3}$ were plotted against the fractional conversion in the figure. The vertical bars represent the range of error in $\lambda_{3}$ on the basis of the averaged values of $\lambda_{3}$, which should result from the error of $\pm 3 \%$ in the estimation of the ratio, $[\eta]_{\text {obs }} /[\eta]_{\lambda=0}$.

All plots for each specimen lie within this range. This fact indicates that such an extent of scattering in measured values of $\lambda_{3}$ as shown in Figure 3 cannot be avoided. The averaged values of $\lambda_{3}$ obtained from several runs are in fairly close agreement with those from the kinetic equation. The tendency that $\lambda_{3}$ increases with the increasing fractional conversion is in close agreement with that expected from eq 9. These facts may offer supporting evidence for the previous kinetic treatment. ${ }^{2}$ The branching parameter $\lambda_{3}$ for PVA was estimated to be in the range of $10^{-6}-10^{-5}$ in the present study. Considering the error in $\lambda_{3}$ originating from the 3-\% error in the estimate of the intrinsic viscosity, the lower limit of the estimate of $\lambda_{3}$ might be $10^{-6}$ in the present analysis. The observed value of $\lambda_{3}$ for the sample $\mathrm{H}-1$ polymerized to the conversion of $79.2 \%$ at $90^{\circ} \mathrm{C}$ was $1.6 \times 10^{-5}$ and $M_{w}$ was found to be $3.44 \times 10^{5}$. Therefor, this polymer has 5.5 weight-average long-chain branches per polymer chain. The observed value of $\lambda_{3}$ for $\mathrm{H}-2$ was lower than that for $\mathrm{H}-1$, though $\mathrm{H}-2$ was polymerized to a higher conversion than $H-1$. These observed values of $\lambda_{3}$ for both the samples, however, are considered to be within the range of the scattering suggested in Figure 3.

When the GPC-viscosity method is used for the general purpose of quantitative determination of the long-chain branches in PVA, attention should be paid to the fact that such an extent of error as that shown in Figure 3 might be included in the observed $\lambda_{3}$.

Acknowledgments. The authors are sincerely grateful to Professor M. Kurata of Kyoto University and Professor H. Fujita of Osaka University for their suggestions for the improvement of this paper, and to Associate Professor M. Kamachi of Osaka University for his valuable advise in computation.

\section{REFERENCES}

1. S. Nozakura, Y. Morishima, and S. Murahashi, J. Polym. Sci., Part A-1, 10, 2781 (1972).

2. S. Nozakura, Y. Morishima, and S. Murahashi, J. Polym. Sci., Polym. Chem. Ed., 10, 2853 (1972).

3. E. E. Drott and R. A. Mendelson, J. Polym. Sci., Part A-2, 8, 1361 (1970). 
Long Branching in PVAc and PVA. V.

4. E. E. Drott and R. A. Mendelson, ibid., 8, 1373 (1970).

5. M. Kurata, H. Okamoto, M. Iwama, M. Abe, and T. Homma, Polymer J., 3, 739 (1972).

6. M. Kurata, M. Abe, M. Iwama, and M. Ma- tsushima, ibid., 3, 729 (1972).

7. M. Kurata, J. Soc. Rubber Ind. Japan, 45, 105 (1972).

8. L. M. Hobbs and V.C. Long, Polymer, 4, 479 (1963). 